\title{
A case of acute brachial artery occlusion after transradial coronary intervention
}

\author{
Zhenchi Sang, Huigen Jin, Zongjun Liu \\ Department of Cardiology, Pu Tuo Hospital, Shanghai University of Traditional Chinese Medicine, Shanghai, China
}

Postep Kardiol Inter 2013; 9, 2 (32): 184-186

DOI: $10.5114 / p w k i .2013 .35457$

\begin{abstract}
In 2010, a 49-year-old man was admitted to our hospital with chest pain. Angiography via the radial approach was performed. Acute brachial artery occlusion was present after the procedure. By transcatheter thrombolysis, brachial artery occlusion was recanalized. Transcatheter thrombolysis seemed to be effective and safe.
\end{abstract}

Key words: brachial artery occlusion, transcatheter thrombolysis, recanalize.

\section{Introduction}

Artery occlusion can be a complication of transradial coronary angiography or intervention. Radial artery occlusion was usual with an incidence of $2-18 \%$ in the literature [1-4], which should in theory not endanger the viability of the hand because of double blood supply. In contrast, brachial artery occlusion was not reported, but can result in acute hand ischemia, even necrosis. Transcatheter thrombolysis was effective and safe, and can be used to recanalize the artery occlusion [5, 6].

The article first discusses transcatheter thrombolysis; it was effective and safe in the condition of acute brachial artery thrombosis after transradial intervention.

\section{Case report}

A 49-year-old male patient was admitted to our hospital with chest pain that started 3 years prior to admission. He experienced squeezing anterior chest pain on exertion which lasted 4-5 min. He had no other concomitant symptoms, including syncope and amaurosis, and no history of hypertension, diabetes or sudden death of family members, with a history of prior smoking for more than ten years. Blood pressure at admission was 120/70 mm Hg. Physical examination revealed a systolic murmur at the left sternal border in the third to fourth intercostal space, with no conduction and tremor, which was more obvious with VALSALVA breathing. A standard 12-lead electrocardiogram showed ST-T wave changes in leads II, III and aVF with high voltage and negative T-waves in leads V1-V5. Echocardiography showed left ventricular hypertrophy, mild to moderate left ventricular outflow tract stenosis with a pressure gradient of $70 \mathrm{~mm} \mathrm{Hg}$, aortic valve calcification, and mild mitral regurgitation. Coronary angiography and left ventriculography via the radial approach were performed to identify. The patient had a positive Allen's test. Local anesthesia was $1 \mathrm{mll}$ of lidocaine $2 \%$ administered $1 \mathrm{~cm}$ below the styloid process of the radius. The right radial artery was punctured with a puncture needle n0.21, and a 0.018" guidewire was introduced, followed by a $6 \mathrm{Fr}$ sheath (TERUMO Co.) $23 \mathrm{~cm}$ long with an external diameter of $2.3 \mathrm{~mm}$. After sheath insertion into the artery, $3000 \mathrm{IU}$ of sodium heparin and a solution of $200 \mu \mathrm{g}$ of nitroglycerin were administered. During catheterization, a 0.035 " guidewire $260 \mathrm{~cm}$ long was positioned in the ascending aorta to exchange the catheters. Our choice was a 5 Fr Tig catheter for the left coronary artery, because it had extreme tortuosity in the right subclavian artery. In case of failure to curve for the right coronary artery, the $5 \mathrm{Fr} \mathrm{Judkins} 4$ curve for the right coronary artery, and a 6 Fr pigtail catheter (CORDIS Co.) for the left ventricular were used. Coronary angiography revealed no significant stenosis. Left ventriculography showed the characteristics of hypertrophic cardiomyopathy; peak pressure gradient in the left ventricular outflow tract was $50 \mathrm{~mm} \mathrm{Hg}$ by cardiac catheterization. After the end of the procedure, the sheath was removed slowly and progressively with monumental flow release through the radial artery.

\section{Corresponding author:}

Dr. Zongjun Liu, Department of Cardiology, Pu Tuo Hospital, Shanghai University of Traditional Chinese Medicine, No. 164, LanXi Road, Shanghai, China 200062, tel.: +86 $02162572723^{\star} 8132$, e-mail: sangzc@126.com

Received: 10.01.2013, accepted: 22.04.2013. 


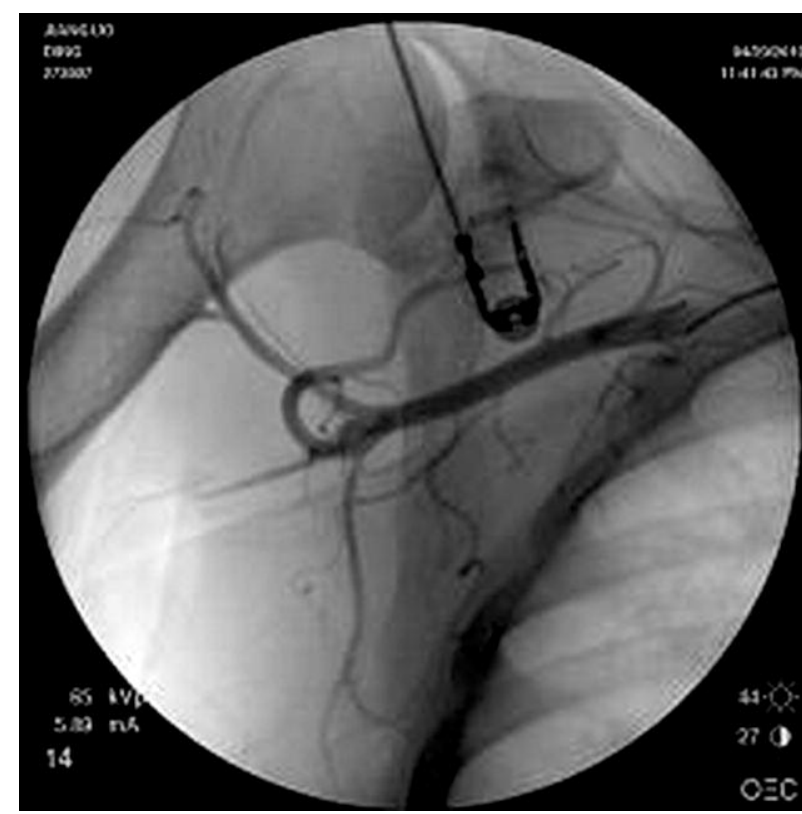

Fig. 1. Angiography showed acute thrombosis of brachial artery

Hemostasis was achieved by application of occlusive tourniquets (Terumo TR Band) with gradual release of pressure for 6-8 $\mathrm{h}$. Approximately $12 \mathrm{~h}$ after the diagnostic procedure, the patient complained of severe right upper limb swelling, pain, pallor, paresthesia, and pulselessness. The distal skin of the right upper limb was hypothermic and pale. The right radial and ulnar pulse had disappeared. The ultrasonographic examination demonstrated no flow of the right radial artery distal to the puncture site and thrombus in the

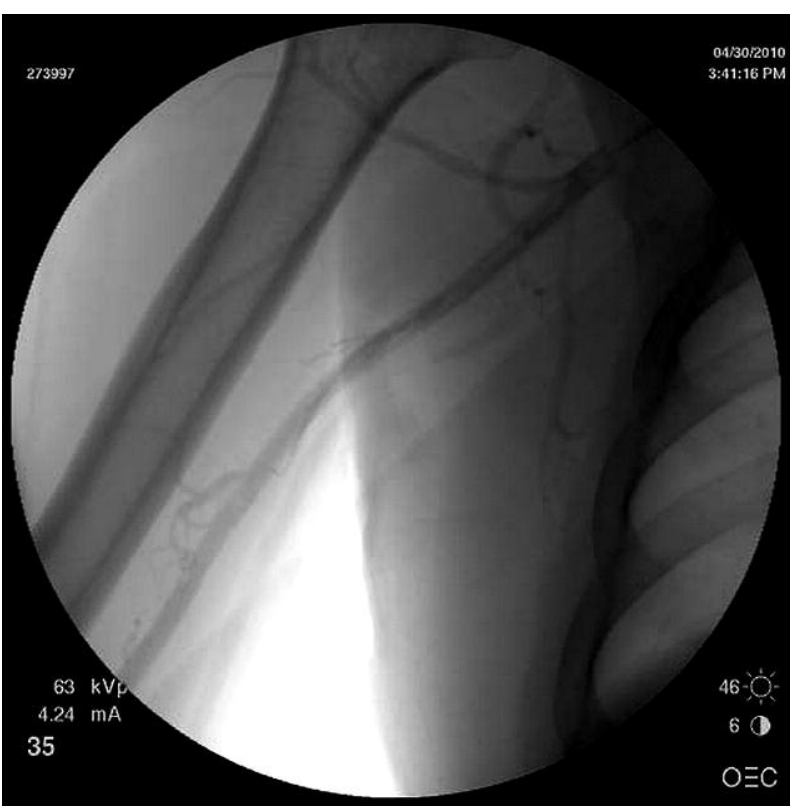

Fig. 3. Blood flow was smooth after thrombolysis in angiography

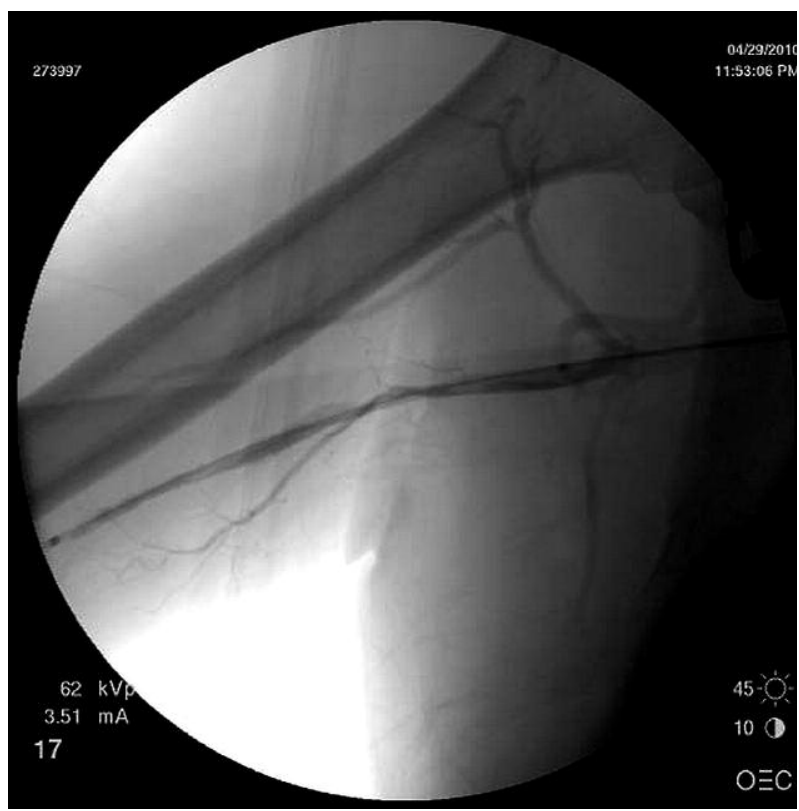

Fig. 2. Thrombolytic catheter into the thrombus

right brachial artery. Considering acute ischemia of the right upper limb, time of onset $<3$ days, we decided to treat with transcatheter thrombolysis. The catheter (YASHIRU) was placed in the right proximal axillary artery via the right femoral artery. Angiography showed decreased flow at the junction of the right brachial artery and the axillary artery, and disappeared flow in the distal brachial artery. Considering acute thrombosis in the right brachial artery (Figure 1), a super smooth guidewire was delivered through the occlusive

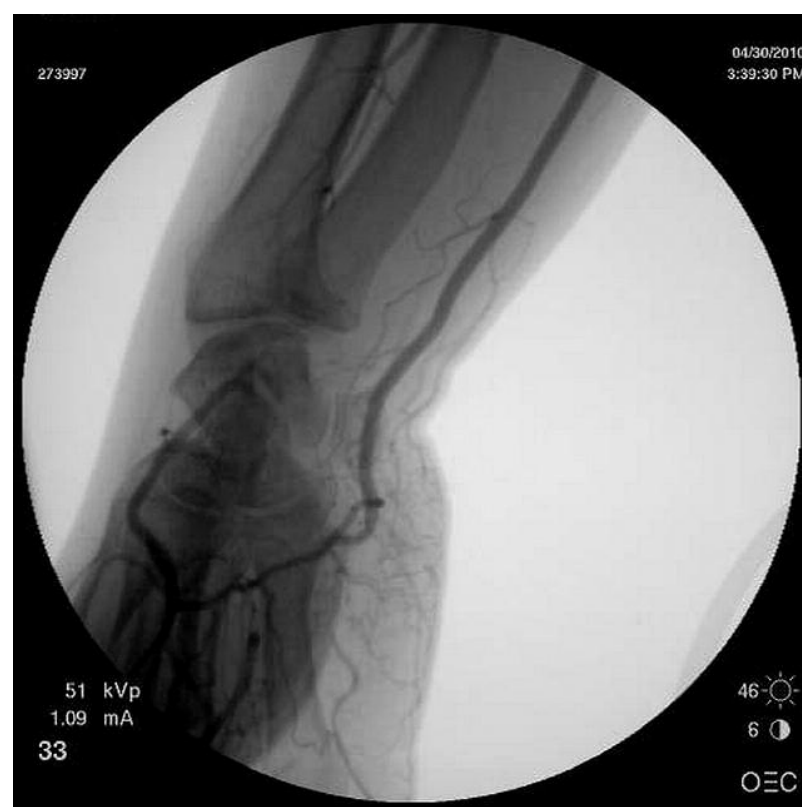

Fig. 4. Palmar arch was present 
thrombus, then placed the thrombolytic catheter (UniFuse) via the wire to the thrombus (Figure 2), continuous infusion of urokinase into the thrombus via minipump for $12 \mathrm{~h}$ with $80,000 \mathrm{U} / \mathrm{h}$, thrombus disappeared, blood flow was smooth in right upper limb arteries after thrombolysis in angiography (Figure 3), and palmar arch was present (Figure 4).

\section{Discussion}

The transradial approach is known as a convenient method for percutaneous coronary angiography with fewer bleeding complications and improved patient's comfort. However, thrombotic occlusion of the radial artery can occur as a complication of this procedure. The relative factors of occlusion might be the dosage of heparin during the procedure, the size and type of sheath, and the duration of pressure. Thrombotic occlusion of the vessel should in theory not endanger the viability of the hand if a double blood supply through the ulnar arch is present. Some patients showed spontaneous recanalization of radial artery occlusion in the follow-up period. In a study, Stella recorded that in 563 patients, 30 patients (5.3\%) had clinical evidence of radial artery occlusion at discharge, but at 1-month follow-up, persistent occlusion was found only in 16 patients (2.8\%) [7]. In the ACCESS study, the incidence of acute radial artery occlusion was $5 \%$, while after 1 month the incidence was only $3 \%$ [8]. However, there was no previous report of brachial artery occlusion after cardiac catheterization in the literature. Because the separation of the brachial artery into the ulnar artery and the radial artery, supplying the forearm, brachial artery occlusion can lead to acute ischemia and necrosis.

In this case, the patient showed clinical evidence of brachial artery occlusion, confirmed by angiography. Thrombotic occlusion was relative to endothelial injury of the vessel in the procedure. Persistent brachial occlusion had clinical sequelae such as necrosis of the hand and even amputation.

The most commonly used treatments include: (1) intravenous thrombolysis, (2) embolectomy via Fogarty catheter, (3) surgical embolectomy, (4) transcatheter thrombolysis $[5,6]$. Our choice was transcatheter thrombolysis because of its minimally invasive, thrombolytic effect, and fewer complications [9]. Especially for fresh thrombus, efficiency was better than old thrombosis. If a guide wire passed through the occlusion, the rate of successful thrombolysis was $92 \%$ [10].

Vascular endothelial injury resulting in acute brachial artery thrombosis was a possible complication of the transradial approach. Although acute brachial artery thrombosis after transradial intervention was rare, it had serious consequences. Therefore careful maneuvering of the catheter was warranted during the transradial approach. In addition, the use of thrombolytic agents via the catheter seemed to be effective and safe in conditions of acute brachial artery thrombosis after transradial intervention.

\section{Acknowledgments}

We acknowledge the contributions of Dr Wei Yang, Dr Weiqing Wang, and catheter laboratory directors of the contributing centers.

\section{References}

1. Sanmartin M, Gomez M, Ramon JR, et al. Interruption of blood flow during compression and radial artery occlusion after transradial catheterization. Catheter Cardiovasc Interv 2007; 70: 185-189.

2. Pancholy S, Coppola J, Patel T, Roke-Thomas M. Prevention of radial artery occlusion-patent hemostasis evaluation trial (PROPHET Study): a randomized comparison of traditional versus patency documented hemostasis after transradial catheterization. Catheter Cardiovasc Interv 2008; 72: 335-340.

3. Pancholy SB. Impact of two different hemostatic devices on radial artery outcomes after transradial catheterization. J Invas Cardiol 2009; 21: 101-104.

4. Cubero JM, Lombardo J, Pedrosa C, et al. Radial compression guided by mean artery pressure versus standard compression with a pneumatic device (RACOMAP). Catheter Cardiovasc Interv 2009; 73: 467-472.

5. Muller Hulsbeck S. Percutanous therapy of occlusions of calf arteries with newly developed hydrodynamic thrombectomy catheter Angiojet LF140. Rofo Fortschr Geb Rontgenstr Neuen Bildgeb Verfahr 1998; 168: 514-517.

6. Ansel GM. Rheolytic thrombectomy in the management of limb ischemia: 30-day results from a multicenter registry. J Endovasc Ther 2002; 9: 395-402.

7. Stella PR, Kiemeneij F, Laarman GJ, et al. Incidence and outcome of radial artery occlusion following transradial artery coronary angioplasty. Cathet Cardiovasc Diagn 1997; 40: 156-8.

8. Mont'Alverne Filho JR. Comparative study of the use of diltiazem as an antispasmodic drug in coronary angiography via the transradial approach. Arq Bras Cardiol 2003; 81: 59-63, 54-8.

9. Ouriel K, Glair DG. Complication associated with the use urokinase and recombinant tissue plasminogen activator for catheter directed peripheral arterial and venous thrombolysis. J Vasc Intrv Radiol 2000; 11: 295-298.

10. Shortell CK, Ouriel K. Thrombolysis in acute peripheral arterial occlusion: predictors of immediate success. Ann Vasc Surg 1994; 8: 59-65. 